\title{
CONCEPTUAL DESIGN AND IMPLEMENTATION OF AUTOMATIC INSPECTION STATION FOR THE WELD QUALITY VISUAL TESTING
}

\author{
P. Kurp*, G. Witkowski**
}

\begin{abstract}
Visual testing is a basic method of weld quality inspection, performed by appropriately trained inspectors. In justified cases - such as, primarily, high volume production - a person can be replaced by a machine. This paper discusses the assumptions of the automatic station for visual testing. It also presents the design of the station and a prototype constructed according to the design. Initial tests of the device have been conducted and the tests results are discussed in the article.
\end{abstract}

Keywords: automation, visual testing, robotics, laser scanning, welding, surface topography

\section{Introduction}

Visual testing (VT) is a basic method applied for the inspection of the weld quality in welded structures of all kinds. The examination covers the weld face and root sides (if accessible) as well as the heat affected zone and the parent material. The tests consist in a thorough inspection of the surface of the welded joints and the surrounding area. In particular, they are used to detect surface discontinuities such as cracks, porosity, delamination, incomplete penetration, concave face and root, craters, excessive weld reinforcement or convexity, burn-throughs, spatter, uneven weld bead etc. All the detected unacceptable discrepancies are described in the quality control chart which provides the basis for classifying the product into one of the three categories: good quality, defective to be repaired or rejected.

Visual tests (VT) are performed by appropriately trained inspectors with the application of tools and in the conditions specified in the above-cited standards. Staff training and experience are nowadays indispensable. As a result of the technological development and the emergence of advanced and precise optical cameras, it is becoming possible to construct an automatic station to conduct this type of tests. The proposed solution is particularly useful in the manufacturing plants with automatic welding lines and stations, where the initial assessment of the weld quality can be performed during or immediately after the welding process.

This article presents the design assumptions of a robotic station for visual testing of the weld quality using an industrial robot KUKA KR-15 equipped with a 3D MicroEpsilon LLT 2800-100 optical head. The paper discusses the stages of designing the station such as the initial assumptions, the selection of the necessary system elements, the simulation of the system operation and the construction of the complete workstation. The article presents the innovative approach to the issue of designing with the application of modern programming techniques.

Piotr Kurp, DSc.: Kielce University of Technology, Department of Industrial Laser Systems, Aleja Tysiąclecia Państwa Polskiego 7, 25314 Kielce, PL, pkurp@tu.kielce.pl

** Grzegorz Witkowski, MSc.: Kielce University of Technology, Department of Automation and Robotic, Aleja Tysiąclecia Państwa Polskiego 7, 25314 Kielce, PL, gwitkowski@tu.kielce.pl 


\section{Station design and its implementation}

Developing a robotic control station involved the application of the rapid prototyping method permitting an immediate creation of the station design. The available engineering tools such as SolidWorks and LabVIEW package were deployed for this purpose. These tools make it possible to simulate the device operation and to eliminate those configurations that may cause collision or undesirable behavior of the system without its physical movement. The virtual scene that was created using software graphics corresponded to the actual conditions of the device operation. The scene included the following elements of the environment: the KUKA KR-15 robot manipulator, the scanning head, the measuring table.

A six-degree-of-freedom manipulator with an open kinematic chain consisting of class $\mathrm{V}$ rotary kinematic pairs was applied.Thanks to the robot kinematics it is possible to manipulate the scanning head in the joint, Cartesian and in the tool configurations. This is especially useful while scanning complex shape multi-surface components.

The MicroEpsilon LLT 2800-100 device is the scanning head. It operates according to the principle of optical triangulation. The laser beam line scans the workpiece surface through the linear optical system. The scattered reflected light is amplified by the CMOS sensor that is a part of the high precision optical system. The image on the CMOS sensor is evaluated in two dimensions, i.e. in the $\mathrm{X}$ and $\mathrm{Z}$ axes as shown in Figure 1. The standard measuring range in the $X$ axis is $50 \mathrm{~mm}$, and in the $Z$ axis $-245 \mathrm{~mm}$. The linearity of the device in both axes is equal to $0.2 \%$. The resolution in the $\mathrm{X}$ axis is 1024 points per profile. The scanning head is controlled by a dedicated controller permitting the establishment of a communication with a PC-class computer for the acquisition and processing of measurement data. The ETHERNET and FireWire interfaces are used for the communication. In the case of the discussed station the second communication standard was applied. By using protocols RS232 and RS44 the controller also has the option to remotely trigger the laser beam and to be synchronized with external devices, such as controllers of external drive axes, or, as in the case of the discussed system, the industrial robot controller.

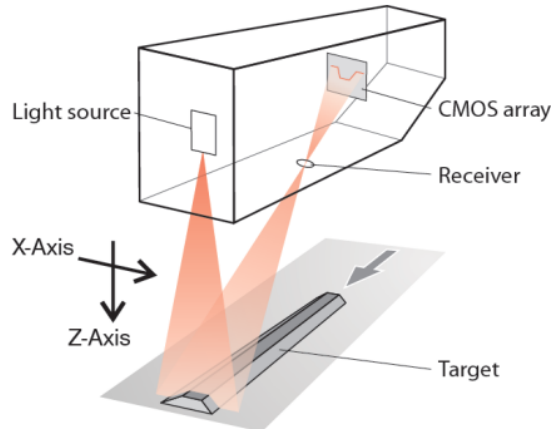

Fig. 1: The principle of scanning the examined element.

Solid models of all the elements of the designed system were developed with the application of the SolidWorks software. The maximum operation range of the industrial robot with the attached scanning head was determined. The possibilities of scanning elements with different surface areas and complex cross-section profiles were analyzed sequentially. Figure 2 present the simulation view of the workstation operation. After the positive verification of the designed station its physical implementation was started.

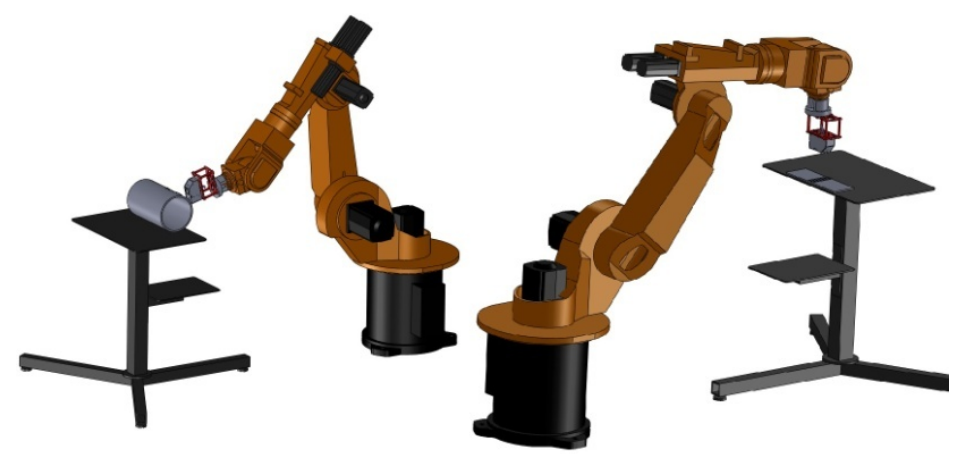

Fig. 2: Different robot manipulator configurations for the scanning task (SolidWorks). 
The physical implementation of the designed workstation involved the use the industrial robot located in the Robotics Laboratory of the Department of Automation and Robotics at the Kielce University of Technology. Using the designed and fabricated attachment member the scanning head was fastened to the flange on the sixth segment of the manipulator. The next stage was to define the effector geometry to determine the tool center point (TCP). The constructed measurment workstation is presented in Figure 3.

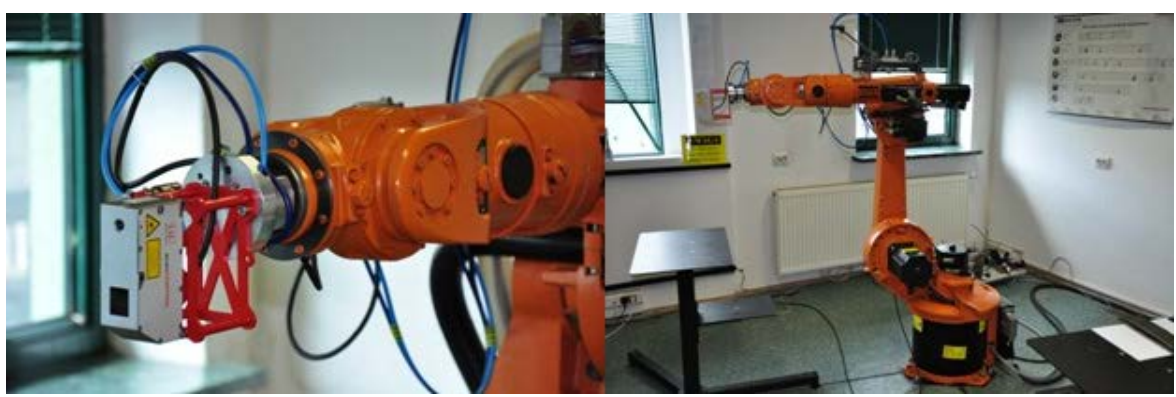

Fig. 3:Kuka KR-15 robot with scanning head attached.

\section{Measurement workstation software}

The standard supplied software for the optical head controller has relatively poor analysis capabilities. The software of the presented device does not permit the imaging of the surface topography of the analyzed workpiece. The lack of such a possibility hinders the inspection of welds. The applied device allows the measurements in the $\mathrm{Z}$ and $\mathrm{X}$ axes. In order to visualize the topography of the tested workpiece one should perform measurements in the third axis. For this purpose the drive for the $\mathrm{Y}$ axis should be deployed. In the proposed automatic workstation the movement in the $\mathrm{Y}$ axis is performed through the KUKA KR-15 robot manipulator.

With the possibility of moving the measuring head in the Y-axis and with the API libraries for the LabView system supplied by the manufacturer of the head, it became possible to record the profiles of the workpieces with the predetermined time step. The recorded data were saved as a series of two dimensional arrays for each axis.

The process of the measurement data acquisition is followed by the process of generating spatial 3D graphs illustrating the scanned surface. The capabilities of the applied programming/software language allow the advanced manipulation of the generated graphs. The view of the part of developed measurement code and research workstation is presented in Figure 4.

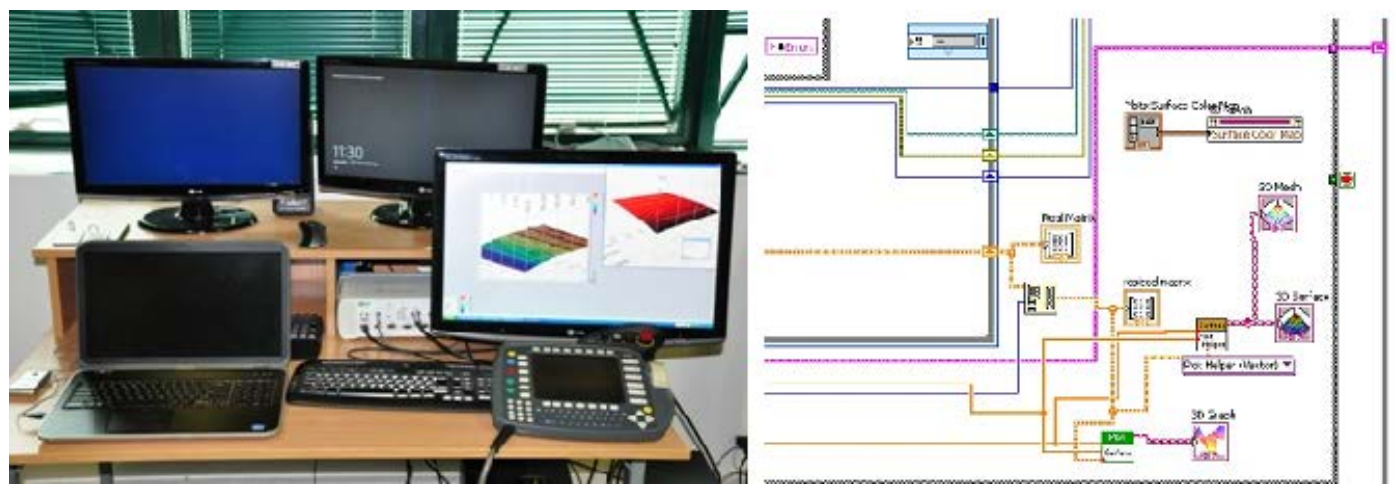

Fig. 4:Prototype research workstation and part of LabView code.

\section{Testing the designed system}

In order to examine the validity of the designed system two experiments were conducted, which concerned the verification of the obtained data for different shapes of the scanned workpieces.

For this purpose the scanning focused on the workpieces in the form of a steel sheet and a steel pipe with penetration welds formed on their surface. The first experiment consisted in scanning to obtain an image of a steel sheet surface topography with three parallel penetration welds. The resultant images of the scanned surface are shown in Fig. 5, which presents both the full view and the close-up of the section. 


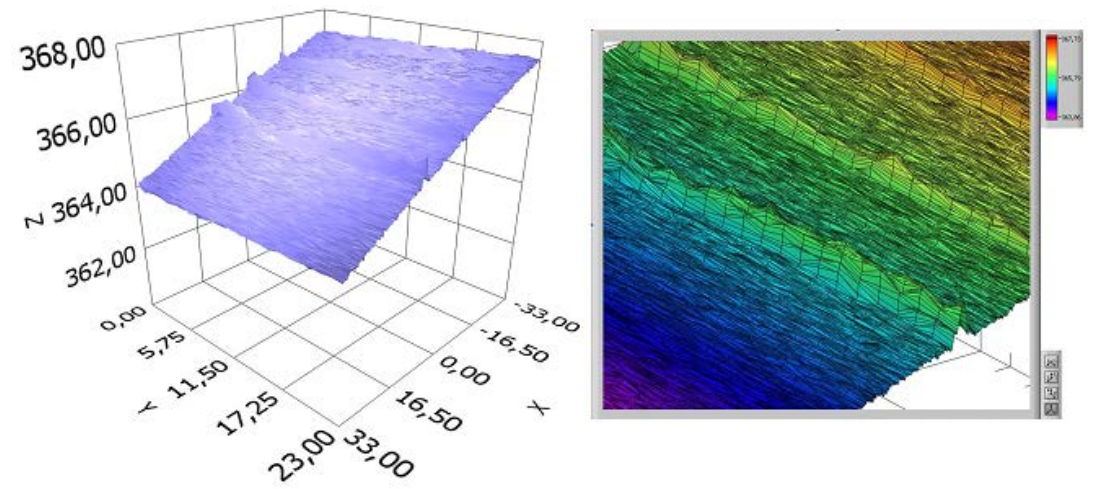

Fig. 5: Topography of the scanned flat surface of the steel sheet with three penetration welds.

The second experiment consisted in scanning to get an image of a topography of the steel pipe surface with a circular pad weld. The resultant images of the scanned surface are shown in Fig. 6, which presents both the full view and the close-up of the section.

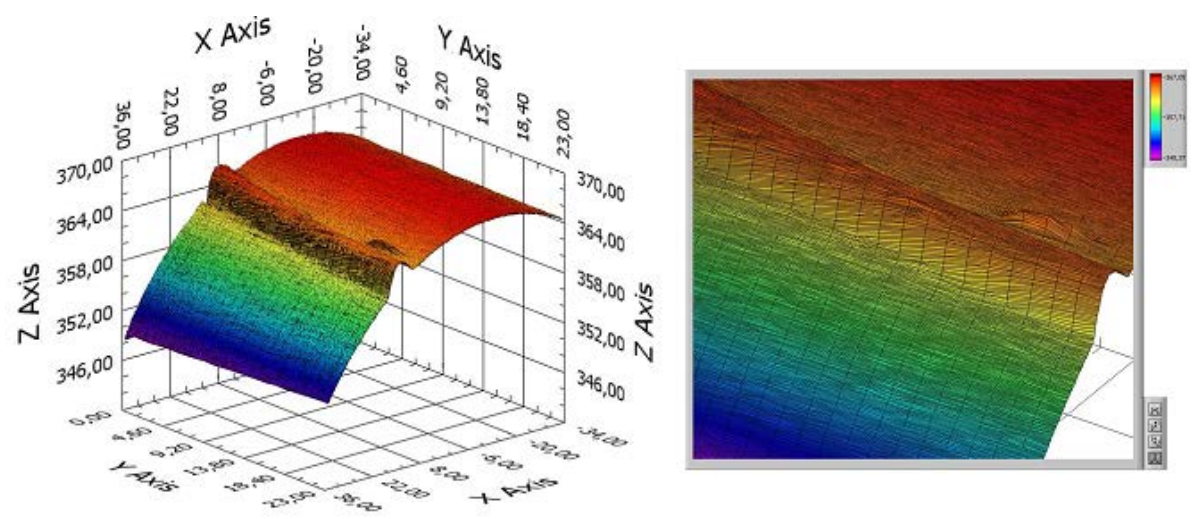

Fig. 6: Topography of the scanned surface of a pipe segment with a pad weld.

\section{Conclusions}

Designed and constructed in the Kielce University of Technology, station based on the use of the KUKA KR-15 industrial robot permits topography visualization of the scanned workpiece surface. The workspace is typical of an anthropomorphic robot, which makes it possible to scan a workpiece with the scanning head being set in specific positions. With six degrees of freedom of the manipulator one can scan the inside of a workpiece, e.g. a pipe with a sufficiently large diameter. The next stage of developing the research station will involve the preparation of algorithms to enable an accurate measurement of the obtained topography, especially of welds. Developing the solutions of recording the obtained topography as a surface in popular standards of 3D data exchange i.e. step or iges will allow a remote observation and inspection.

\section{References}

PN-EN ISO 9712:2012 Non-destructive tests - Qualification and certification of NDT personel [in Polish].

PN-EN ISO 17637:2011 Non-destructive tests of welds - Visual tests of welded joints [in Polish].

PN-EN ISO 17635:2010 Non-destructive tests of welds - General rules concerning metals.

PN-EN ISO 5817:2014-05 Welding - Welded joints in steel, nickel, titanium and their alloys (with the exception of beam welded joints) - Quality levels according to welding discrepancies [in Polish].

PN-EN 13018:2004 Non-destructive tests - Visual tests - General rules.

PN-EN 13927:2009 Non-destructive tests - Visual tests - Equipment.

Nozdrzykowski K., Janecki D. (2014) Comparative studies of reference measurements of cylindrical surface roundness profiles of large machine components. Metrology and Measurement Systems: XXI(2014), Warsaw, pp. 67-76.

Szcześniak A., Szcześniak Z. (2009) Methods and analysis of processing signals of incremental optoelectronic transducer. REVIEW OF SCIENTIFIC INSTRUMENTS: Vol. 80(2009), pp. 1-16. 